
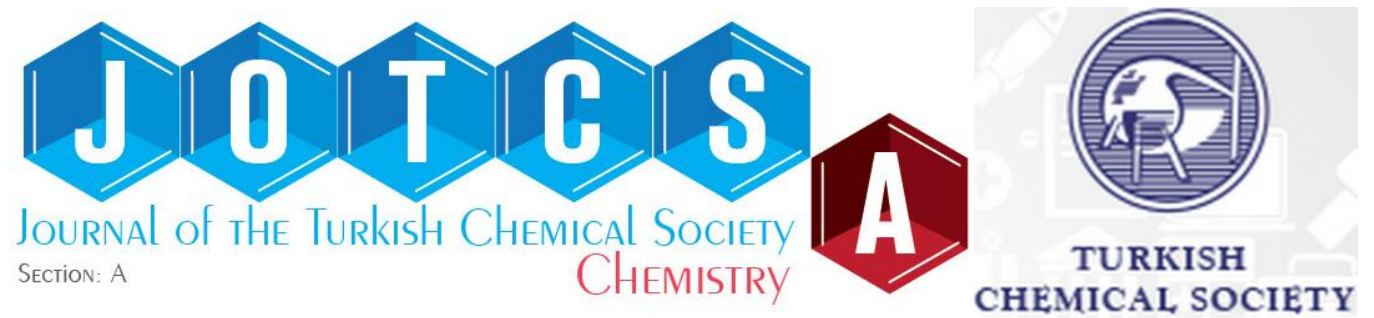

\title{
Study on the Synthesis and Characterization of Antibacterial Polystyrenes
}

\author{
Gokhan Acik ${ }^{1 *}$ ID \\ ${ }^{1}$ University of Piri Reis, Faculty of Science and Letters, Department of Chemistry, TR-34940, Istanbul, \\ Turkey.
}

Abstract: In the present work, a series of quaternized antibacterial polystyrenes (anti-PSts) with various molecular weights is synthesized and characterized. Initially, $\omega$-bromo end functional polystyrenes with different molecular weights (PSt-Br-, PSt-Br-2 and PSt-Br-3) are synthesized by atom transfer radical polymerization (ATRP). Then, the $\omega$-bromo functionalities of obtained PSt-Brs are reacted with trimethylamine (TEA) to achieve corresponding antibacterial properties. PSt-Brs and anti-PSts produced are structurally characterized by Fourier transform infrared spectroscopy (FT-IR), proton nuclear magnetic resonance spectroscopy ( $\left.{ }^{1} \mathrm{H}-\mathrm{NMR}\right)$, and gel permeation chromatography (GPC) at various stages. Glass transition temperatures $\left(T_{\mathrm{g}}\right)$ of PSt-Brs and anti-PSts are determined by differential scanning calorimetry (DSC) analysis. Water contact angle measurement (WCA) is employed for the wettability characterization studies of anti-PSts. In addition, antibacterial activity of final products against to Gram-positive (Staphylococcus aureus) and Gram-negative (Escherichia coli) bacteria is elucidated in terms of molecular weight and quaternization reaction time.

Keywords: antibacterial properties, quaternization, polystyrene.

Submitted: April 01, 2019. Accepted: May 12, 2019.

Cite this: Acik G. Study on the Synthesis and Characterization of Antibacterial Polystyrenes. JOTCSA. 2019;6(2):245-52.

DOI: https://dx.doi.org/10.18596/jotcsa.547471.

*Corresponding author. E-mail: (gacik@pirireis.edu.tr)

\section{INTRODUCTION}

Polystyrene (PSt) as one of the oldest and one of the major synthetic thermoplastic polymers in use today, finds in countless commercial applications, commonly applied in food contact packaging, medical, construction, electronic and housings material industries due to its insulating and physical properties and low-cost (1-5). Despite the its outstanding properties including acceptable mechanical performance, good chemical inertness and excellent processing capability, technological advances and environmental and energy concerns in the field have given rise to the necessity of introducing additional features to polystyrene, for example improved electrical conductivity, biodegradability, mechanical and antibacterial properties. Thus, several strategies have been devoted to modify the properties of PSt. Kiatkamjornwong and co- workers prepared starch-g-polystyrene copolymers to achieve higher biodegradability for PSt (6). Park et al. worked on mixing PSt with organophilic clay in order to enhance the mechanical properties of polystyrene (7). Thermal conductivity of PSt was improved, preparing polymer composite having a matrix of polystyrene containing aluminium nitride reinforcement by $\mathrm{Yu}$ and co-workers (8). The electrical conductivity of PSt was increased by McNally and co-workers, preparing multiwalled carbon nanotube/polystyrene composite (9). On the other hand, up to now, the synthetic methods for well-defined block, graft, star shaped, hyperbranched, brush-like copolymers possessing polystyrene building block via various well-known polymerization techniques have been published in the literature for a wide range of applications (10-15). 
Significant research efforts have been currently invested by researchers to improve the antibacterial activity of a wide variety of polymers or their derivatives against microorganisms. There are mainly three types of compounds known to be antibacterial in literature: quaternary phosphonium salts, pyridinium salts and quaternary ammonium salts $(16,17)$. Among them, polymeric materials bearing quaternary ammonium group (QAS) that covalently bonded to active sides of polymer chains have outstanding importance for various fields of polymer science and industry due to its high charge density and excellent processability (18).

Copper-based atom transfer radical polymerization (ATRP) is one of the most effective and widely used methods of controlled radical polymerization. This robust method which proceeds through a redox equilibrium between $\mathrm{Cu}(\mathrm{I})$ and $\mathrm{Cu}(\mathrm{II})$ species allows the utilizing a large variety of radically polymerizable monomers, brings many advantages by providing control over molecular weight, molecular weight distribution and chain end functionalities, and can be triggered with a wide range of halogenated initiators or macro-initiators (19). Furthermore, it is worth mentioning that the $\omega$-halogen at the end-group of polymers obtained by ATRP can be readily converted into other functional groups such as double bonds, amines or azides by chemical transformations (20).

Herein, we reported the successful syntheses of anti-PSts via ATRP followed by direct quaternization reaction with TEA. The main objectives of the present research were the determination of antibacterial properties of antiPSts having different molecular weights $\left(M_{\mathrm{n}}, \mathrm{GPC}=\right.$ 2300,3400 and $5700 \mathrm{~g} . \mathrm{mol}^{-1}$ ) and reacted with TEA for different time intervals (24, 48 and $72 \mathrm{~h}$ ). anti-PSts with higher molecular weight or obtained by long quaternization time significantly inhibited both the growth of $S$. aureus and $E$. coli $(\mathrm{p}<0.05)$ compared to others and neat PSt-Br as well as control samples.

\section{EXPERIMENTAL SECTION}

\section{Materials}

Styrene (St; 99\%, Sigma Aldrich, Steinheim, Germany) used as monomer and was passed through a basic alumina column to remove the inhibitor prior to use. $N, N, N^{\prime}, N^{\prime \prime}, N^{\prime \prime}-$ pentamethyldiethylenetriamine (PMDETA; 99\%, Sigma Aldrich, Steinheim, Germany) was used as ligand and distilled prior to use. Copper(I) bromide (CuBr; 98\%, Sigma Aldrich, Steinheim, Germany) was used as catalyst and used as received. Ethyl-2-bromopropionate (EtBrP; $>99 \%$, Sigma Aldrich, Steinheim, Germany) was used as initiator and used as received. Triethylamine (TEA; $\geq 99 \%$ ) was purchased from Sigma Aldrich (Steinheim, Germany). Rectangular glass slices with dimensions of $76 \times$ $26 \mathrm{~mm}$ used were supplied from ISOLAB
(Istanbul, Turkey) and all other solvents were purchased from Sigma Aldrich (Steinheim, Germany) and used without further distillation.

\section{Characterizations}

Fourier transform infrared (FT-IR) spectra were recorded on a Perkin-Elmer brand FTIR Spectrum One spectrometer equipped with a diamond attenuated total reflection (ATR) device scanning range covered $400 \mathrm{~cm}^{-1}$ to $4000 \mathrm{~cm}^{-1}$ (Lambda 25, Waltham, USA). ${ }^{1} \mathrm{H}-\mathrm{NMR}$ spectra were recorded on a Varian 600 Spectrometer operating at $600 \mathrm{MHz}$ in chloroform-d with tetramethylsilane as internal standard. Gelpermeation chromatography (GPC) analyses were attained from a ViscotekGPC max Autosampler system containing a pump module (GPCmax, Viscotek Corp., Houston, TX, U.S.A.), a combined light scattering (Model 270 Dual Detector, Viscotek Corp.), and a refractive index (RI) detector (VE 3580, Viscotek Corp.). The light scattering detector $\left(\lambda_{0}=670 \mathrm{~nm}\right)$ included two scattering angles: $7^{\circ}$ and $90^{\circ}$. The RI-detector was calibrated versus polystyrene standards having narrow molecular weight distribution, and so, the quoted molecular weights of the polymers are expressed in terms of polystyrene equivalents. Two columns, that is, a $7.8 \times 300$ $\mathrm{mm}$ LT5000L, Mixed, Medium Org and a $7.8 \times$ 300 mm LT3000L, Mixed, Ultra-Low Org column equipped with a guard column $4.6 \times 10 \mathrm{~mm}$ (Viscotek, TGuard), were used for the chloroform eluent at $35^{\circ} \mathrm{C}$ (flow rate: $1 \mathrm{~mL} \mathrm{~min}^{-1}$ ). Data were analyzed using ViscotekOmniSEC Omni-01 software. Thermal properties were evaluated by TGA and DSC analyses using a SeikoSII TG-DTA 6300 TG/DTA analyzer heated from 20 to $800^{\circ} \mathrm{C}$ with a heating speed of $10{ }^{\circ} \mathrm{C} / \mathrm{min}$ and a DSC analyzer (Mettler Toledo DSC) with the samples (3-5 $\mathrm{mg}$ ) sealed in aluminium pans using heating rate of $10^{\circ} \mathrm{C} / \mathrm{min}$ in the temperature ranging from 25 to $200{ }^{\circ} \mathrm{C}$ under nitrogen atmosphere, respectively. WCA measurements were conducted by a KSV Attension Theta Optical Tensiometer (CAM-200, Vastra Frolunda, Sweden) under laboratory conditions using grade water for chromatography.

\section{General Procedure for Atom Transfer Radical Polymerization (ATRP) and Sequential Quaternization Reaction}

Firstly, $\omega$-bromo polystyrenes with various molecular weights were synthesized as described elsewhere by ATRP (11). Briefly, styrene $(5 \mathrm{~mL}$, $44 \mathrm{mmol})$, PMDETA ( $90 \mu \mathrm{L}, 0.44 \mathrm{mmol}), \mathrm{CuBr}(62$ $\mathrm{mg}, 0.44 \mathrm{mmol})$, EtBrP $(56 \mu \mathrm{L}, 0.44 \mathrm{mmol})$, and deoxygenated toluene $(8 \mathrm{~mL})$ were mixed in a Schlenk tube equipped with a magnetic stirring bar. The mixture was degassed by three freezepump-thaw cycles, left under vacuum, and placed in an oil bath at $110{ }^{\circ} \mathrm{C}$ for $30 \mathrm{~min}$. After end of the polymerization time, the reaction mixture was diluted with tetrahydrofuran (THF) and then passed through a short column of neutral alumina to get rid of the copper salt. The excess THF was rotary-evaporated under vacuum. The $\omega$-bromo 
PSt was dissolved in a small amount of THF, and precipitated in cold methanol twice. The final product was dried under reduced pressure at room temperature for $24 \mathrm{~h}$ and labelled as PSt$\mathrm{Br}-1$. A representative quaternization reaction procedure between $\mathrm{PSt}-\mathrm{Br}-1$ and TEA was as follows: A mixture of PSt-Br-1 $(1 \mathrm{~g}, 0.4 \mathrm{mmol})$ and TEA $(60 \mu \mathrm{L}, 0.4 \mathrm{mmol})$ in anhydrous THF $(8 \mathrm{~mL})$ were prepared, argon gas was passed through for $5 \mathrm{~min}$ and heated at $50{ }^{\circ} \mathrm{C}$ for $24 \mathrm{~h}$. After the reaction time, the excess THF was evaporated and the quaternized polymer was purified by two precipitations in cold methanol and this polymer is labelled as anti-PSt-1. PSt-Br-2 and PSt-Br-3 or anti-PSt-2 and anti-PSt-3 were synthesized under similar experimental conditions for different time intervals. The various data of the obtained PStBrs and anti-PSts were summarized in Table 1.

\section{Polymeric coating procedure}

The obtained anti-PSts were coated onto a rectangular glass slice with dimensions of $76 \times 26$ $\mathrm{mm}$ by means of a precise mechanical dip-coater. Typical coating procedure was as follows: Firstly, anti-PSts were dissolved by THF in a $50 \mathrm{~mL}$ beaker to achieve $\sim 20 \mathrm{mg} / \mathrm{mL}$ concentration of solutions. Then, glass slices, which were washed with chromic acid and distilled water, dipped into the prepared solutions to be coated by withdraw rate of $90 \mathrm{~mm} / \mathrm{min}$ and $1 \mathrm{~min}$ of waiting duration. The coated glass slices were maintained in a fume hood so as to get rid of the residual THF for 72 hours at room temperature prior to WCA measurements.

\section{Contact angle measurement}

The contact angle between surfaces of coated glass slices with anti-PSts and a water drop were measured by sessile drop technique using a micro-syringe with a stainless steel needle. The captured images of water drop on the sample surfaces by a conventional digital camera were taken four times in order to indicate the reproducibility of the data. The reported contact angles were the mean value of these measurements.

\section{Antibacterial test of PSt-Brs and anti-PSts}

The bacterial culture of S. aureus ATCC 25923 and E. coli ATCC 25922 were grown on nutrient agar slants and stored at $4{ }^{\circ} \mathrm{C} .15 \mathrm{~mL}$ tryptic soy broth (TSB) in a sterile centrifuge tube, including a loop full of bacteria from the agar slant, was incubated $35{ }^{\circ} \mathrm{C}$ for $24 \mathrm{~h}$. The diluted to a concentration of $10^{6} \mathrm{CFU} / \mathrm{mL}$ the bacterial cultures were used as a test suspension for the antibacterial activity tests. The glass slices coated with the PSt-Br and anti-PSt samples were cut into $1 \times 1 \mathrm{~cm}$ coupons to be used for antibacterial activity test. Then the coupons were transferred into $15 \mathrm{~mL}$ sterile tubes and $2 \mathrm{~mL}$ of the test suspension was added on each tube and incubated at $35^{\circ} \mathrm{C}$ for $24 \mathrm{~h}$ in a shaking incubator. After $24 \mathrm{~h}$ of contact time, serial dilutions from each sample were spread inoculated on plate count agar (PCA) plates. The PCA plates were incubated at $35{ }^{\circ} \mathrm{C}$ for $24 \mathrm{~h}$ and colonies were counted. Colonies were counted manually. As a general rule for plate counting, only the petri dishes with 30 to 300 colonies were used for counting. The number of colonies was calculated based on the dilution factor of the counted petri dishes. The experiments were replicated twice. Three different coupons were prepared for each of the samples. Then they were transferred into separate test tubes and inoculated with the test suspensions. Data were expressed as means \pm standard deviation for the three replicates. Differences between the means were analysed using Student's t-test, taking $\mathrm{p}<0.05$ as statistically significant.

\section{RESULTS AND DISCUSSION}

The $\omega$-halogen terminated polymers are of significant academic and industrial interests because they can be easily transformed into useful functionalities by means of either electrophilic addition reactions or nucleophilic displacement reactions to achieve various macromolecular architecture having unique chemical properties. Mainly, there are two methodologies for the synthesis of QAS containing polymers in the literature; (i) direct polymerization of monomers possessing QAS, or its copolymerization with other monomers and (ii) direct quaternization of polymers possessing either tertiary phosphonium/ammonium groups or alkyl halides. In the first strategy, a limiting effect of monomeric stability and steric hindrance on the degree of quaternization can be mentioned. In our work, a series of bromine endfunctional polystyrenes with different molecular weights were synthesized by ATRP initiated by EtBrP, using CuBr/PMDETA catalytic system. The obtained polystyrenes with bromine end-groups were reacted with TEA in THF to obtain antibacterial polystyrenes for different time intervals. The synthetic strategy for the preparation of PSt-Brs having different molecular weight bearing quaternary ammonium salt is represented in Scheme 1.

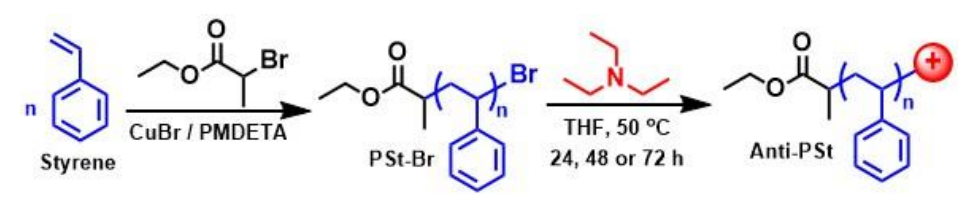

Scheme 1: Schematic representation of preparing process for anti-PSts. 
Table 1: The various data of the obtained PSt-Brs and anti-PSts.

\begin{tabular}{|c|c|c|c|c|c|c|}
\hline Sample & $\begin{array}{c}\text { Time } \\
\text { (hour) }\end{array}$ & Conv. $(\%)^{c}$ & $\begin{array}{c}M_{\mathrm{n}}^{\mathrm{d}} \\
\left(\mathrm{gr} \cdot \mathrm{mol}^{-1}\right)\end{array}$ & $\oplus^{d}$ & $\begin{array}{c}D Q^{e} \\
(\mathrm{~mol} \%)\end{array}$ & $\begin{array}{c}T_{g}^{f} \\
\left({ }^{\circ} \mathrm{C}\right)\end{array}$ \\
\hline PSt-Br-1 ${ }^{a}$ & 0.5 & 25 & 2300 & 1.19 & - & 101.6 \\
\hline PSt-Br-2a & 1 & 34 & 3400 & 1.21 & - & 104.5 \\
\hline PSt-Br-3a & 1.5 & 39 & 5700 & 1.18 & - & 108.2 \\
\hline anti-PSt- $1^{b}$ & 24 & 87 & 2650 & 1.20 & 24.8 & 103.5 \\
\hline anti-PSt- $2^{b}$ & 24 & 92 & 4050 & 1.24 & 25.5 & 109.5 \\
\hline anti-PSt-3ab & 24 & 88 & 6550 & 1.18 & 29.5 & 112.0 \\
\hline anti-PSt-3b & 48 & 90 & 6850 & 1.20 & 34.3 & 114.4 \\
\hline anti-PSt- $3 c^{b}$ & 72 & 90 & 7100 & 1.18 & 41.0 & 118.1 \\
\hline
\end{tabular}

${ }^{a}[\mathrm{M}] /[\mathrm{I}] /[\mathrm{CuBr}] /[\mathrm{PMDETA}]: 100 / 1 / 1 / 1$; temperature: $110{ }^{\circ} \mathrm{C} .{ }^{b}[\mathrm{PSt}-\mathrm{Br}] /[\mathrm{TEA}]: 1 / 1$; temperature: $50{ }^{\circ} \mathrm{C}$. ${ }^{c}$ Conversions were determined gravimetrically. ${ }^{d}$ Molecular weights and $\mathrm{Ds}$ were determined by GPC analysis. ${ }^{e}$ The quaternization degree was determined by ${ }^{1} \mathrm{H}-\mathrm{NMR}$ analysis using Equation $1 .{ }^{\text {TT }}$ The glass transition $\left(T_{\mathrm{g}}\right)$ temperature determined by DSC analysis.

The FT-IR spectra of PSt-Brs with different molecular weight synthesized by ATRP were shown in Figure 1 to evidence their structures. As can be seen from the FT-IR spectra of PSt-Brs, the characteristic absorption bands of the stretching vibrations of aliphatic and aromatic C$\mathrm{H}$ at around $3100-2800 \mathrm{~cm}^{-1}$ range, $\mathrm{C}=\mathrm{C}$ stretching vibrations at 1605 and $1490 \mathrm{~cm}^{-1}$, weak aromatic overtone and combination bands in the 1940 to $1680 \mathrm{~cm}^{-1}$ region, $-\mathrm{CH}_{2}$ bending vibration at $1450 \mathrm{~cm}^{-1}$, and $\mathrm{Y}(\mathrm{C}-\mathrm{H})$ from aromatic ring at around $755 \mathrm{~cm}^{-1}$ proved the success of ATRP $(10,11)$. On the other hand, after quaternization reaction the characteristic peaks appeared at around $1155-1067 \mathrm{~cm}^{-1}$ region assigned to quaternary ammonium groups confirmed the formation of desired anti-PSts (Figure 2) $(18,21)$.

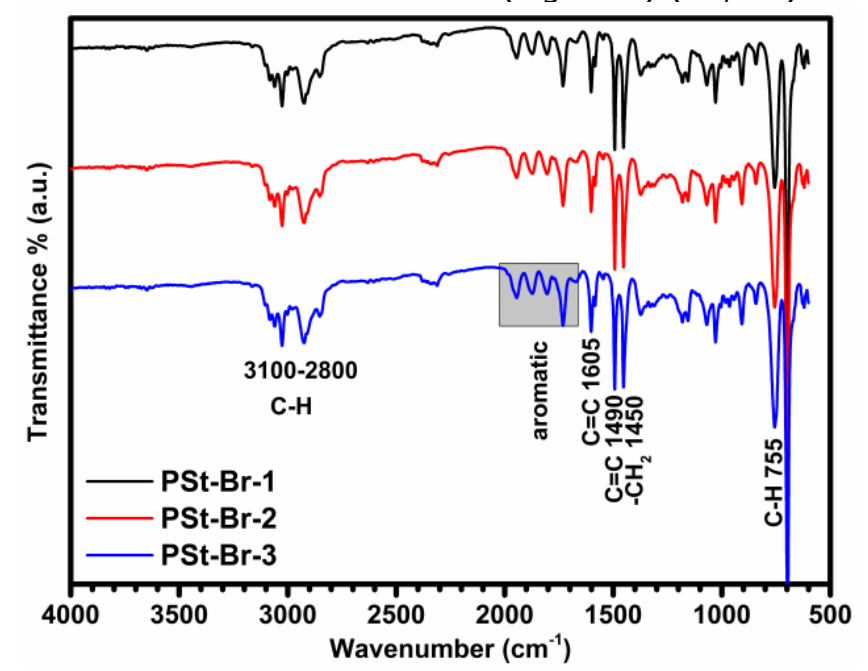

Figure 1: FT-IR spectra of PSt-Brs with different molecular weights synthesized by ATRP.

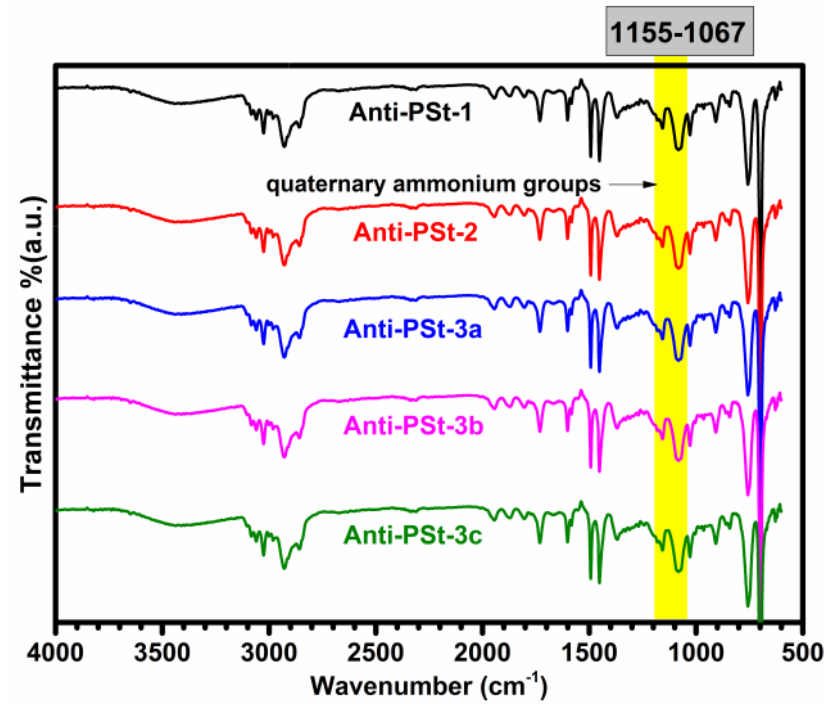

Figure 2: FT-IR spectra of anti-PSts obtained by reaction PSt-Brs with TEA.

The ${ }^{1} \mathrm{H}$-NMR studies were also carried out for additional confirmation of the syntheses of PSt-
Brs and anti-PSts (Figures 3 and 4). As illustrated in Figures 3 and 4, the resonances related to the 
methine proton close to the bromine chain end were appeared at around 4.27-4.47 ppm (c) and quantitatively shifted to $3.75 \mathrm{ppm}$ as a result of nucleophilic displacement of the bromine atom at the polymer chain end by a TEA. In addition, the quaternization reaction was followed by ${ }^{1} \mathrm{H}-\mathrm{NMR}$ study. As can be seen in the Figure 4, after the quaternization reaction, the characteristic methyl protons of TEA attached to chain end were detected at around 0.90 and 3.15 ppm ( $f$ and e). Also, the increased peak intensity of the $f$ and $e$ protons of TEA with increased time intervals indicated a higher degree of quaternization. The degree of quaternization (DQ) was calculated by utilizing the peak areas of the aliphatic $-\mathrm{CH}_{2}$ protons (a) and aliphatic protons of TEA ( $f$ and e) using Equation 1 and achieved data were given in Table 1.

$$
\mathbf{D Q} \%=\left[\frac{\mathrm{e} / 2+\mathrm{f} / 3}{\mathrm{e} / 2+\mathrm{f} / 3+\mathrm{a} / 2}\right] \times 100
$$

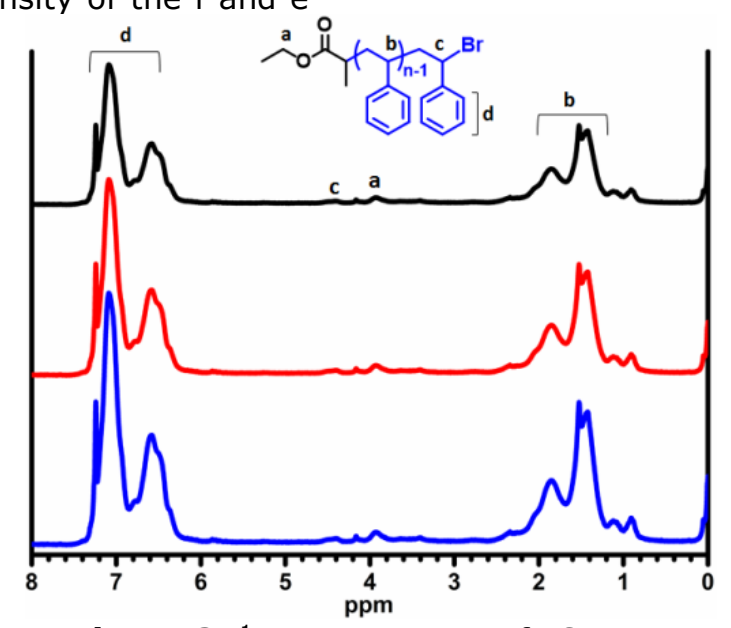

Figure 3: ${ }^{1} \mathrm{H}-\mathrm{NMR}$ spectra of PSt-Brs.

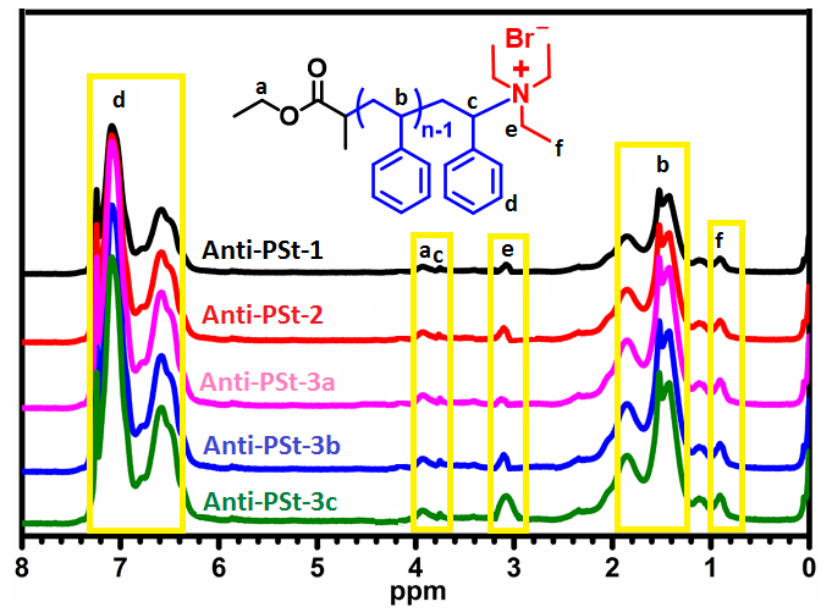

Figure 4: ${ }^{1} \mathrm{H}-\mathrm{NMR}$ spectra of anti-PSts.

The comparative GPC traces of PSt-Brs and antiPSts were as presented in Figures 5 and their molecular weights and polydispersity indexes ( $Ð$ ) were summarized in Table 1 . The unimodal GPC traces with low molecular weight distribution, which trend the shifted to higher molecular weight without homopolymer formation, were observed for PSt-Br-1, PSt-Br-2 and PSt-Br-3 in line with the ATRP reaction time interval. In Figure 5, one may notice the slightly increased molecular weights after quaternization reaction. On the other hand, when the anti-PSt-3a, antiPSt-3b and anti-PSt-3c were evaluated among themselves, it was clear that the polymers obtained as the quaternization time increases tend to shift to higher molecular weights. It was convenient to show these results as chromatographic evidence of quaternization reactions conducted. 




Figure 5: GPC chromatograms of PSt-Brs and anti-PSts.

Thermal behaviors of obtained polymers were investigated by differential scanning calorimetry (DSC). The DSC thermograms of PSt-Brs and anti-PSts were shown in Figure 6 and the glasstransition temperatures $\left(T_{\mathrm{g}}\right)$ were tabulated in Table 1 . The PSt-Br-3 sample had a relatively high $T_{\mathrm{g}}$ value compared to PSt-Br-2 and PSt-Br-1 probably due to its higher molecular weight which caused to increase in molecular attractions or restriction of molecular motion. There was also an increasing trend in $T_{\mathrm{g}}$ value from anti-PSt-3a to anti-PSt-3c owing to the increase in the degree of quaternization depending on quaternization time. This increasing trend might be due to the an decrease in chain mobility by increased ionic attraction among the polymer chains $(21,22)$.

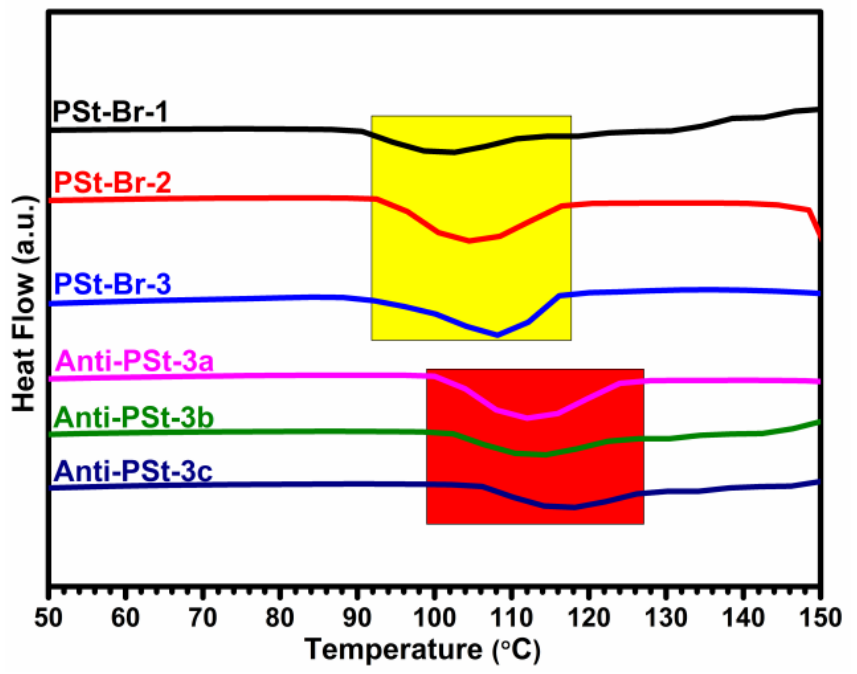

Figure 6: DSC thermograms of PSt-Brs and anti-PSts.

Figure 7 have indicated the contact angle determined between PSt-Br-1, PSt-Br-2, PSt-Br3, anti-PSt-3a, anti-PSt-3b and anti-PSt-3c surfaces and a water drop (WCA), and also shows the captured images of a water drop on those samples in order to estimate the effect of molecular weight and DQ on the wettability properties of the polymers achieved. Flat polystyrene (PSt) is known as a hydrophobic polymer in the literature (23). In our case, the
WCA values of PSt-Brs increased from $90^{\circ} \pm 1$ to $98^{\circ} \pm 1$ probably due to the increasing molecular weight resulted from longer polymer main chain. Conversely, the WCA value of anti-PSt-3 decreased gradually from $86^{\circ} \pm 1$ (anti-PSt-3a) to $77^{\circ} \pm 1$ (anti-PSt-3c) with an increase of DQ in good agreement with the literature studies (24, 25 ). The slight differences in the contact angles determined also provided supporting evidence that anti-PSts were successfully prepared. 


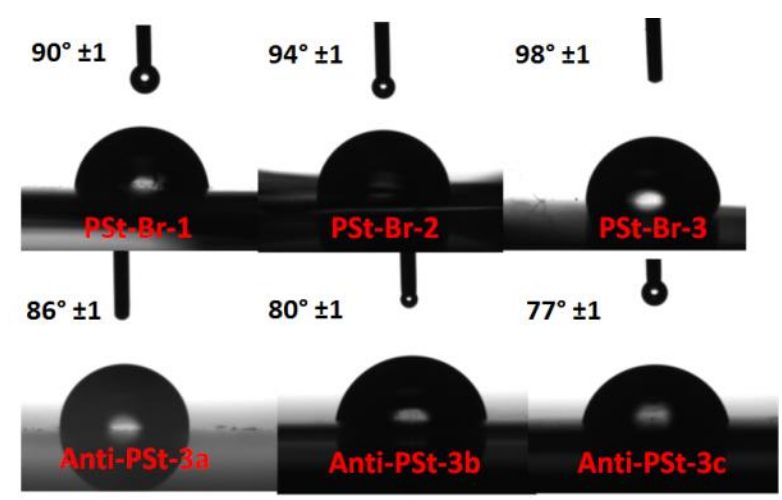

Figure 7: Contact angle images for droplets water on surfaces coated with PSt-Brs and anti-PSts.

The obtained PSt-Brs by ATRP with various molecular weights were chemically modified to produce quaternary ammonium salts in the presence of TEA for different intervals of time so as to improve their antibacterial activity against to gram negative and gram positive bacteria. For this purpose, the $S$. aureus and E.coli were utilized to determine the antibacterial property of PSt-Br and anti-PSt discs by direct contact test (incubation for $24 \mathrm{~h}$ at $35{ }^{\circ} \mathrm{C}$ ). The anti-PSt coupons inhibited both the growth of $S$. aureus and E.coli significantly $(\mathrm{p}<0.05)$ compared to the

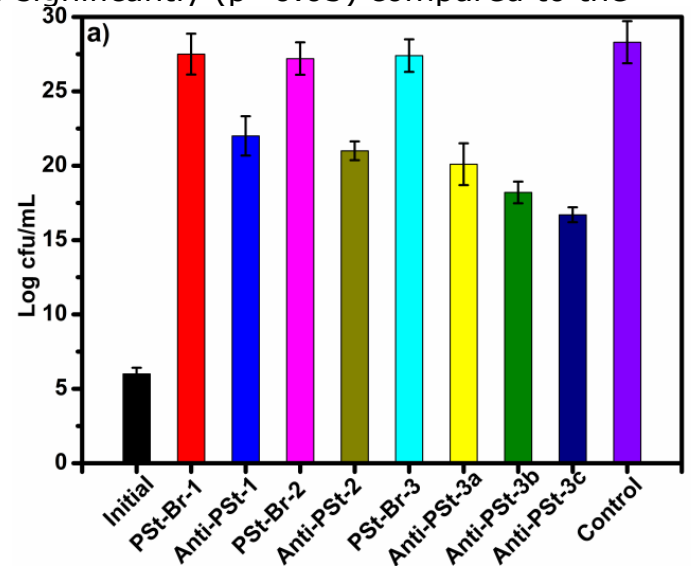

Figure 8: Antibacterial activity against (a) S. aureus, (b) E. coli after $24 \mathrm{~h}$ of incubation at $35^{\circ} \mathrm{C}$. (Initial; initial bacterial count, Control; incubated medium without sample).

\section{CONCLUSIONS}

In conclusion, anti-PSts with different DQ ranging from 24.8 to $41.0 \mathrm{~mol} \%$ and with different molecular weights from 2650 to $7100 \mathrm{~g} \cdot \mathrm{mol}^{-1}$ were successfully synthesized by quaternization of PSt-Br obtained through ATRP with TEA for different intervals of time under mild conditions. The successful formations of anti-PSts and their precursors was verified by the spectral (FT-IR and $1 \mathrm{H}-\mathrm{NMR})$, chromatographic (GPC), thermal (DSC) and wettability (WCA) analyses. Antibacterial tests showed that the anti-PSts significantly inhibited both the growth of the $S$. aureus and E.coli. As a result, anti-PSts can be particularly useful in the fabrication of food contact packaging and medical supplies in near future.

\section{CONFLICT OF INTEREST}

The authors declare that there is no conflict of interest regarding the publication.

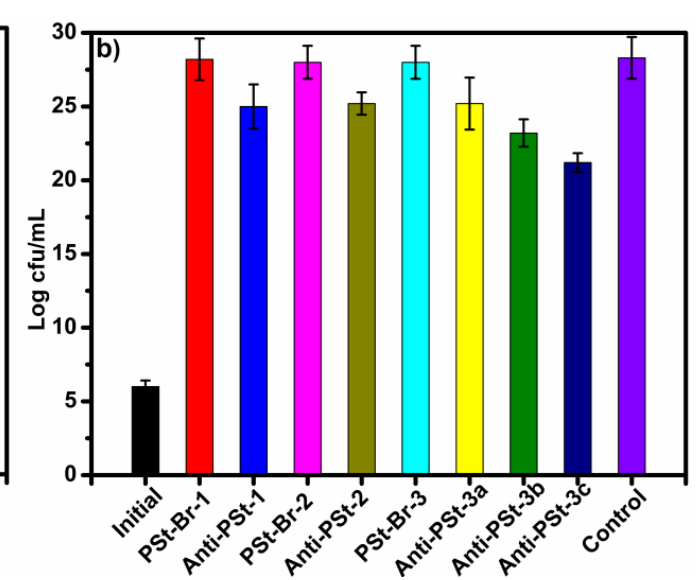

PSt-Br and control samples (Figures $8 \mathrm{a}$ and $8 \mathrm{~b}$ ). Almost no reduction in $S$. aureus and E.coli counts of PSt-Br samples was determined, whereas more than 5 log reduction (>99.99\%) was observed for the $S$. aureus counts, and about 4 log reduction (99.9\%) was observed for $E$. coli counts of the anti-PSts. Besides, anti-PSt-3c having both highest molecular weight and $D Q$ depending on reaction times inhibited the growth of $S$. aureus and E.coli more than the other samples, as expected.

\section{REFERENCES}

1. Jin Y, Li Z, Yang L, Xu J, Zhao L, Li Z, et al. Porous aromatic framework 48/gel hybrid material coated solid-phase microextraction fiber for the determination of the migration of styrene from polystyrene food contact materials. Analytical chemistry. 2017; 89(2): 1290-8.

2. Liu W, Nie M, Wang Q. Polybutene-1 tube with in situ microfibering polystyrene via helical convergent flow: an economical pathway to continuously fabricate biaxially reinforced polyolefin tubes for medical application. RSC Advances. 2014; 4(88): 47793-6.

3. Chen W, Hao H, Hughes D, Shi Y, Cui J, Li Z-X. Static and dynamic mechanical properties of expanded polystyrene. Materials \& Design. 2015; 69: $170-80$. 
4. Schmidt P, Cioffi M, Voorwald HJC, Silveira J. Flexural test on recycled polystyrene. Procedia Engineering. 2011; 10: 930-5.

5. Lawania K, Sarker P. Global warming implications of the use of by-products and recycled materials in western Australia's housing sector. Materials. 2015; 8(10): 6909-25.

6. Kiatkamjornwong S, Sonsuk M, Wittayapichet S, Prasassarakich P, Vejjanukroh P-C. Degradation of styrene-g-cassava starch filled polystyrene plastics. Polymer Degradation and Stability. 1999; 66(3): 323-35.

7. Park CI, Park OO, Lim JG, Kim HJ. The fabrication of syndiotactic polystyrene/organophilic clay nanocomposites and their properties. Polymer. 2001; 42(17): 7465-75.

8. Yu S, Hing $P, H u X$. Thermal conductivity of polystyrene-aluminum nitride composite. Composites Part A: applied science and manufacturing. 2002; 33(2): 289-92.

9. McClory C, Pötschke P, McNally T. Influence of Screw Speed on Electrical and Rheological Percolation of Melt-Mixed High-Impact Polystyrene/MWCNT Nanocomposites. Macromolecular Materials and Engineering. 2011; 296(1): 59-69.

10. Kahveci MU, Acik G, Yagci Y. Synthesis of Block Copolymers by Combination of Atom Transfer Radical Polymerization and Visible LightInduced Free Radical Promoted Cationic Polymerization. Macromolecular rapid communications. 2012; 33(4): 309-13.

11. Acik G, Kahveci MU, Yagci Y. Synthesis of block copolymers by combination of atom transfer radical polymerization and visible light radical photopolymerization methods. Macromolecules. 2010; 43(21): 9198-201.

12. Zhang Z, Chalkova E, Fedkin M, Wang C, Lvov SN, Komarneni $S$, et al. Synthesis and characterization of poly (vinylidene fluoride)-gsulfonated polystyrene graft copolymers for proton exchange membrane. Macromolecules. 2008; 41(23): 9130-9.

13. Muchtar Z, Schappacher M, Deffieux A. Hyperbranched nanomolecules: regular polystyrene dendrigrafts. Macromolecules. 2001; 34(22): 7595-600.

14. Schappacher M, Deffieux A. New polymer chain architecture: synthesis and characterization of star polymers with comb polystyrene branches.
Macromolecules. 2000; 33(20): 7371-7.

15. Rzayev J. Synthesis of polystyrenepolylactide bottlebrush block copolymers and their melt self-assembly into large domain nanostructures. Macromolecules. 2009; 42(6): 2135-41.

16. Xue $\mathrm{Y}$, Xiao $\mathrm{H}$, Zhang $\mathrm{Y}$. Antimicrobial polymeric materials with quaternary ammonium and phosphonium salts. International journal of molecular sciences. 2015; 16(2): 3626-55.

17. Tiller JC, Lee SB, Lewis K, Klibanov AM. Polymer surfaces derivatized with poly (vinyl-Nhexylpyridinium) kill airborne and waterborne bacteria. Biotechnology and bioengineering. 2002; 79(4): 465-71.

18. Gao B, Zhang X, Zhu Y. Studies on the preparation and antibacterial properties of quaternized polyethyleneimine. Journal of Biomaterials Science, Polymer Edition. 2007; 18(5): 531-44.

19. Matyjaszewski K. Atom transfer radical polymerization: from mechanisms to applications. Israel Journal of Chemistry. 2012; 52(3-4): 206-20.

20. Matyjaszewski K, Xia J. Atom transfer radical polymerization. Chemical reviews. 2001; 101(9): 2921-90.

21. Acik G, Altinkok C, Olmez H, Tasdelen MA. Antibacterial film from chlorinated polypropylene via CuAAC click chemistry. Progress in Organic Coatings. 2018; 125: 73-8.

22. Stoica D, Ogier L, Akrour L, Alloin F, Fauvarque J-F. Anionic membrane based on polyepichlorhydrin matrix for alkaline fuel cell: Synthesis, physical and electrochemical properties. Electrochimica Acta. 2007; 53(4): 1596-603.

23. Ilhan $F$, Fabrizio EF, McCorkle L, Scheiman DA, Dass A, Palczer A, et al. Hydrophobic monolithic aerogels by nanocasting polystyrene on amine-modified silica. Journal of Materials Chemistry. 2006; 16(29): 3046-54.

24. Tu Q, Wang J-C, Liu R, He J, Zhang Y, Shen $S$, et al. Antifouling properties of poly (dimethylsiloxane) surfaces modified with quaternized poly (dimethylaminoethyl methacrylate). Colloids and Surfaces B: Biointerfaces. 2013; 102: 361-70.

25. Brown PS, Bhushan B. Mechanically durable liquid-impregnated honeycomb surfaces. Scientific reports. $2017 ; 7(1): 6083$. 